\title{
A curve-shaped flexible mesh cage for treatment of large segmental bone defects - a finite element analysis
}

\author{
Jan Wieding*, Robert Souffrant, Wolfram Mittelmeier, Rainer Bader \\ Department of Orthopaedics, University Medicine Rostock, Rostock, Germany, \\ *corresponding author: jan.wieding@med.uni-rostock.de
}

\begin{abstract}
The repair of large segmental defects in long bones caused by fracture, tumour or infection is still a challenging problem in orthopaedic surgery. Titanium scaffolds are clinically used and show good results in bone healing. At the boneimplant-interface the edge of the scaffold can migrate into the bone due to high compression stress. In the present study a new type of flexible titanium mesh cage was investigated in order to determine the mechanical properties for application in segmental defects. Finite Element Method (FEM) was used to characterize the influence of the cutting design and the number of load-bearing segments on the mechanical properties of the mesh cages. Material properties were extracted from standardized test samples of titanium sheet made of the same material (Titan, Grade 2). The results for all analysed designs revealed a decrease of the mechanical properties with increasing size of the cuts. Structural modulus for cut parameters was within the range of human cortical bone with a maximum of $17.1 \mathrm{GPa}$.
\end{abstract}

\section{Introduction}

Repairing large segmental defects in long bones can still be challenging in orthopaedic surgery. These defects can be the consequence of severe fractures, tumour or infection. Besides autologous and allogenic grafts artificial materials like calcimphosphate ceramics and titanium, implants were successfully used as bone scaffolds. Autologous grafts still are the first-choice-materials because of their high biocompatibility. But their grafting represents a risk of postoperative complications like donor site morbidity [1-3], the need for a second operation and subsequent higher operational costs and a limited availability. Allogenic bone grafts are mass available but are associated with problems of poor mechanical stability and can involve immunological reactions, which may lead to amputation in worst case [4]. Therefore, synthetic materials and stabilization systems have been investigated to compensate these disadvantages. Implants made of titanium are biocompatible and mechanically more stable and are therefore suitable to be placed within large defects in highly loaded areas.

In particular, titanium mesh cages have been successfully used in the past for spinal fusion and stabilization [5-7]. With additionally stabilization these principle can also be used for large segmental bone defects. Reconstruction of large segmental long bone defects with a titanium mesh cage has been initially reported by Cobos et al. [8]. Further in vivo animal testing confirmed the efficiency of using a metallic cage $[9,10]$ and some groups reported the use of titanium cages for the treatment of large segmental bone defects in clinical application [11-13]. They showed good clinical results in bone healing and bone union.

Nevertheless, subsidence of the implant into the adjacent bone or bone fracture can occur due to the small contact area within the bone-implant-interface and the high axial stiffness of the implant and mechanical loading at the in- terface $[14,15]$. Fractures of the mesh cages have also been reported [16].

The majority of these implant systems comprised a cylindrical shape with rhombic cut-outs reinforced with two or more internal rings $[7,14]$. This lead to a stiff implant design compared to the mechanical properties of bone. Furthermore, existing mesh cages exhibit a stiff cylindrical cross-section and cannot be adapted to the shape of the adjacent bone, i.e. the mechanical load cannot be transferred adequately.

The aim of this numerical study was to investigate the axial stiffness of a new design of cylindrical mesh cage by the means of finite element analysis. The new system features a curved-shape cross section. This enlarges the boneimplant-interface and gives the possibility of radial flexibility to be formed individually to the bone defect and to provide high axial stiffness at the same time carrying the mechanical loads. Furthermore, numerical analysis was performed to determine the influence of the cutting design on structural stiffness and compressive strength.

\section{Materials \& Methods}

By means of Finite Element Analysis (FEA), the influence of the geometrical design on the structural behaviour of the cage was determined. Cylindrical tubes with an outer radius of $\mathrm{R}=10 \mathrm{~mm}$ and a height $\mathrm{H}=30 \mathrm{~mm}$ were perforated with different cutting designs and locally expanded in radial direction. Subsequently, plastic deformation of the struts allowed the creation of the curve-shaped cross section, which exhibited a larger bone-implant contact area (Image 1). Furthermore, the scaffold could be formed to cope with the shape of the defect it was placed into it due to the flexibility in radial direction. All models have been meshed with linear hexahedral elements with three elements for the wall thickness of $0.5 \mathrm{~mm}$. 


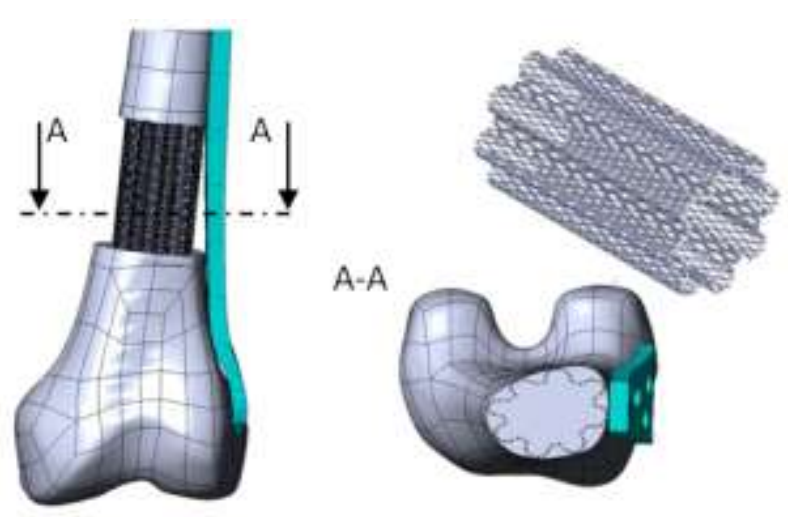

Image 1 Possible application of the curve-shaped mesh cage into a femoral defect adopting to its bone contour.

\section{Material properties}

In order to implement a reliable material behavior with plastic deformation, experimental tensile testing was performed on test samples $(\mathrm{n}=5)$ according to a test standard (DIN 5012512.5 x 50) made of titanium (Grade 2). Data from the tensile testing was implemented as elastic-plastic material properties with isotropic hardening in the $\mathrm{FE}$ Software ABAQUS (Vers. 6.10 EF, Dassault Systèmes, Vélizy-Villacoublay, France).

\section{Parameter variation}

Two parameters of the cuts have been varied, the cut length $1(7.4,7.6,7.8$ and $8.0 \mathrm{~mm})$ and the cut width $\mathrm{b}(0.2$, $0.4,0.6$ and $0.8 \mathrm{~mm})$. Furthermore, the amount of the segments has been increased from 5 (design 1, one quarter of enrolled cylinder is shown in image 2) to 6 (design 2) and 7 (design 3). All other parameters, e.g. width $B=2 \pi R$ resulted from the geometric design. Hence, 48 different cages have been investigated.

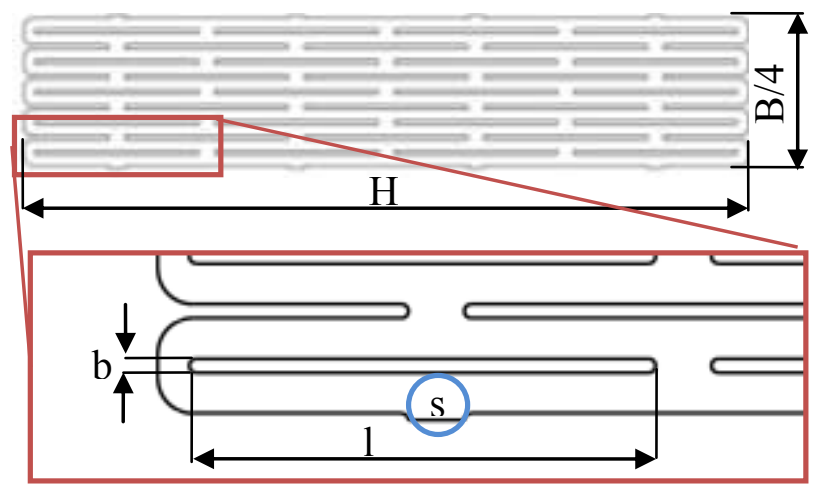

Image 2 Geometrical structure of the cage segments (horizontally orientated). Cut length $l$ and cut width $b$ was varied.

\section{Determination of the expansion forces}

In a first step, uni-axial tensile testing in the circumferential direction was performed on the perforated, enrolled lateral surface of one fourth of the cylinder in order to determine the required forces to enlarge the circumference contour for the curve-shaped cross-section. The symmetry line of the vertical strut junction between two horizontally orientated segments was taken for boundary conditions (marked with an s in image 2). The lower struts were fixed in the vertical direction and a displacement of $\Delta \mathrm{u}=20.2 \mathrm{~mm}$ was applied to the upper struts. This displacement corresponds with one fourth of the circumference contour increase between round and curve-shaped cross-section. The necessary force (reaction force) to widen the segments was analyzed.

\section{Determination of the curve-shaped mechanical behavior} In a second step, radial expansion was performed to create the desired curved cross-shape (Image 3). Due to the symmetry, only one fourth of the cage was modelled. Symmetry boundary conditions were applied to the strut junction of the circular model. Expansion was performed with three rigid bodies (RB) in radial direction. Furthermore, two additional rigid bodies were used as a counter bearing.

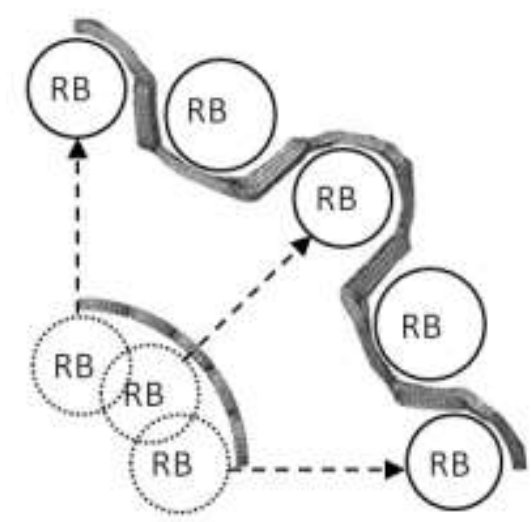

Image 3 Expansion scheme of the rigid bodies (RB) from circular shape to the curved shape.

Subsequently, axial compression testing was performed on the shaped cages until failure occurred or at least for a displacement of $15 \mathrm{~mm}$. For the simulation of the compression testing, two rigid surfaces were used as compression plates. Contact was modelled as frictionless surface to surface contact. Structural properties were calculated from load-displacement relation.

\section{Results}

\section{Material properties}

Apparent stress-strain relation of the tensile testing showed good agreement for the applied material properties between the numerical simulation and experimental testing with deviations less than $3 \%$ for both elastic and plastic regions up to $25 \%$ elongation (Image 4 ).

\section{Expansion forces}

The results of the expansion forces for each of the sixteen numerical models for the three designs with varying number of segments $(5,6$ and 7$)$ are shown in image 5 (top: design 1, middle: design 2, bottom: design 3 ). With increasing length and width of the cuts the expansion force decreased, whereat increasing the cut width had a stronger influence than increasing the cut length. In addition, increasing the number of segments also decreased the expan- 
sion force (design 1, 2 and 3 with 5, 6 and 7 segments, respectively).

For design 3 with 7 segments the cut width of $0.8 \mathrm{~mm}$ was larger than the remaining segment width. Therefore, no results are given in the last column for design 3 in image 5.

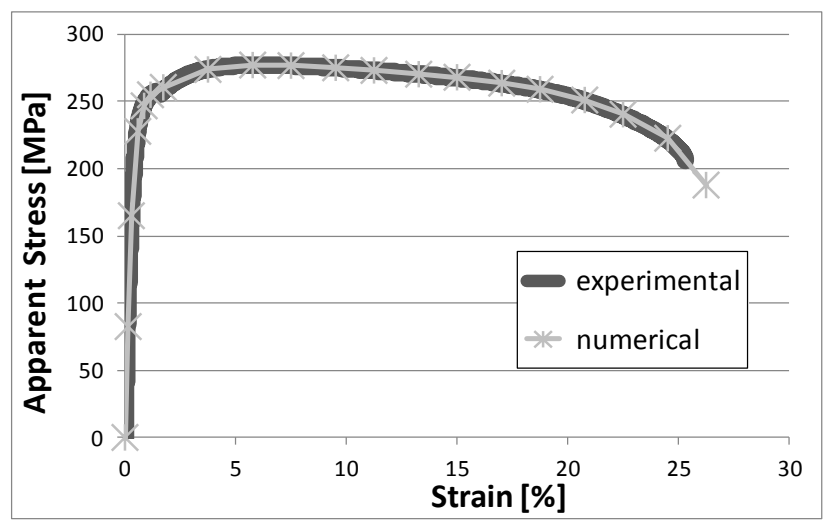

Image 4 Apparent stress-strain relation related to the nominal cross-section for the experimental and numerical tensile test.

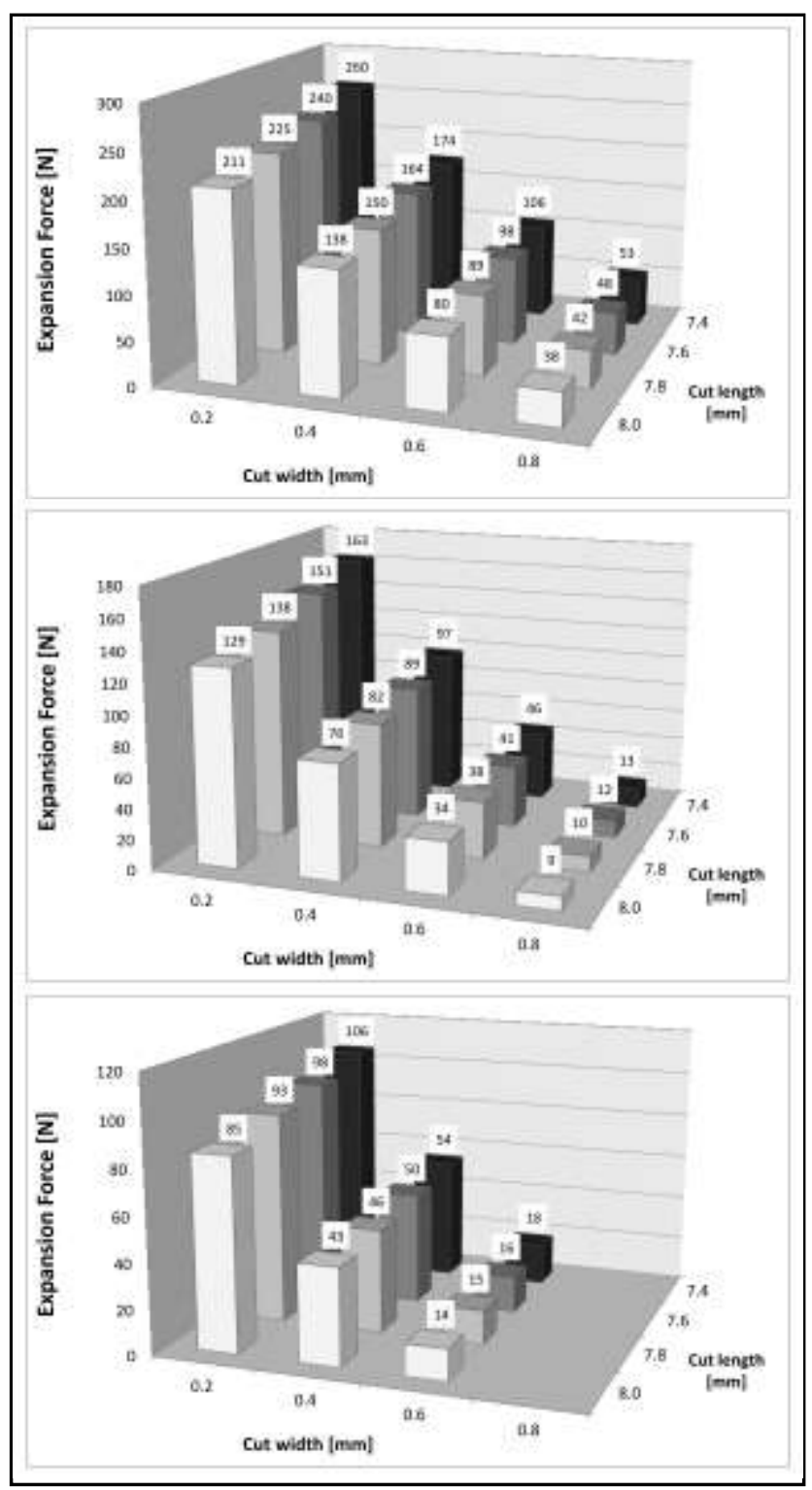

Image 5 Expansion force for the expansion for varying cuts for design 1(top), design 2 (middle) and design 3 (bottom).
Compression behavior of the curve-shaped cage

After expansion and compression the structural modulus was calculated from the slope of the stress-strain relation before failure of the structure occurred (image6).

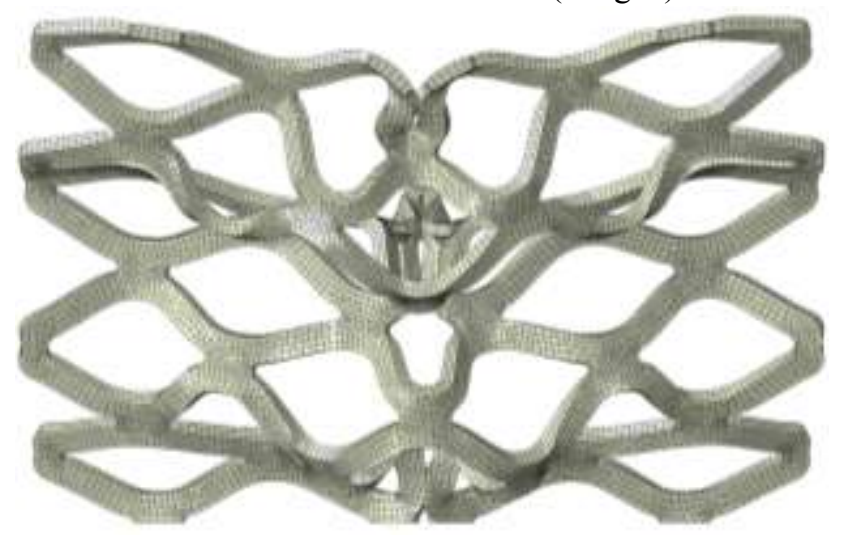

Image 6 Cage deformation (central area) after axial compression.

Due to the complexity of the models, only the basic cut parameter combinations (maximum cut width and length for each design) as indicated by the results of the expansion forces were analyzed and the results of the compression test are listed in Table 1.

Table 1 Structural modulus of the investigated models. All values are given in GPa.

\begin{tabular}{|c|l|l|l|l|l|}
\hline \multicolumn{2}{|c|}{} & \multicolumn{3}{c|}{ Cut width [mm] } & \multirow{2}{*}{} \\
\cline { 2 - 5 } \multicolumn{2}{c|}{} & 0.2 & 0.6 & 0.8 & \\
\hline \multirow{\Xi}{\Xi}{} & 7.4 & $\mathbf{1 3 . 4}$ & n.a. & $\mathbf{3 . 8}$ & \multirow{2}{*}{ Design 1 } \\
\cline { 2 - 5 } & 8.0 & $\mathbf{1 7 . 1}$ & n.a. & $\mathbf{1 . 3}$ & \\
\cline { 2 - 5 } & 7.4 & $\mathbf{1 1 . 0}$ & n.a. & $\mathbf{1 . 8}$ & \multirow{2}{*}{ Design 2 } \\
\cline { 2 - 5 } & 8.0 & $\mathbf{1 4 . 1}$ & n.a. & $\mathbf{0 . 6}$ & \\
\cline { 2 - 5 } & 7.4 & $\mathbf{3 . 4}$ & $\mathbf{1 . 5}$ & n.a. & \multirow{2}{*}{ Design 3 } \\
\cline { 2 - 5 } & 8.0 & $\mathbf{2 . 8}$ & $\mathbf{1 . 1}$ & n.a. & \\
\cline { 2 - 4 }
\end{tabular}

Numerical compression results revealed a structural modulus for the titanium mesh cages between 0.6 and 17.1 GPa. Furthermore, similar to the expansion forces, structural properties were stronger influenced by the width of the cutting design than by the length. Highest elastic modulus could be determined for each design for the model with the thinnest and shortest cuts. Furthermore, increasing the number of segments decreased the mechanical properties.

\section{Discussion}

Modifications of the cutting design showed a high degree of variation possibilities with respect to the adjustment of the targeted mechanical property. Therefore, titanium mesh cages with a structural modulus comparable to human bone [17] as well as cages with lower stiffness can be adapted for any desired application area.

Both, width and length of the cuts revealed an influence on the mechanical properties. Thereby, the width of the cuts had a larger influence.

Increasing the width of the cuts for the models subsequently decreased the width of the remaining struts and there- 
fore accounted for the decrease in the mechanical properties.

A similar influence could be observed for the increase in the number of segments. A larger number of segments implemented, that each segment is less expanded during the expansion of the cage. Therefore, the expansion force decreased with increasing number of segments. At the same time the width of the cuts had a stronger influence because the overall width of each segment was smaller.

Highest structural modulus was found for the mesh cage with the lowest amount of segments, i.e. design 1 with 5 segments and the smallest cut width and length.

For the application as a scaffold for bone regeneration within segmental defects of long bones (e.g. femur), additional stabilization with osteosynthesis systems (e.g. intramedullary nail, plat or fixateur externe) is necessary and subsequently support the load-bearing of the implant.

Hence, the mechanical properties of the cage can be lowered. Furthermore, lower stiffness of the mesh cage may reduce the stress in the bone as predicted by FE analysis for a spinal model [18].

Nevertheless, it has to be considered, that the cage only consisted of a titanium sheet with a wall thickness of $0.5 \mathrm{~mm}$. Increase of wall thickness will therefore, lead to an increase in the mechanical properties.

Furthermore, the cross-section of the cage was nearly expanded by twice the initial diameter (approximately 30 and $15 \mathrm{~mm}$ respectively). Subsequently, the cuts revealed a rhombic shape. Therefore, performing a lower expansion of the cages may reveal a higher axial stiffness compared to the investigated ones.

In the present study we presented the basic concept of a new titanium mesh cage. In future clinical application advantages of this flexible mesh cages can be early weightbearing and mobility as well as the possibility to adapt their cross-sections to that of the bone stock.

\section{References}

[1] Laurie SW, Kaban LB, Mulliken JB, Murray JE. Donor-site morbidity after harvesting rib and iliac bone. Plast Reconstr Surg 1984; 73(6): 933-8.

[2] Younger EM, Chapman MW. Morbidity at bone graft donor sites. J Orthop Trauma 1989; 3(3): 1925.

[3] Wippermann BW, Schratt HE, Steeg S, Tscherne H. [Complications of spongiosa harvesting of the ilial crest. A retrospective analysis of 1,191 cases]. Chirurg 1997; 68(12): 1286-91.

[4] Delloye C, de NP, Allington N, Munting E, Coutelier L, Vincent A. Massive bone allografts in large skeletal defects after tumor surgery: a clinical and microradiographic evaluation. Arch Orthop Trauma Surg 1988; 107(1): 31-41.

[5] Eck KR, Bridwell KH, Ungacta FF, Lapp MA, Lenke LG, Riew KD. Analysis of titanium mesh cages in adults with minimum two-year follow-up. Spine 2000; 25(18): 2407-15.

[6] Payer M. Implantation of a distractible titanium cage after cervical corpectomy: technical experience in 20 consecutive cases, by M. Payer, Volume 148, Issue
11, November 2006, Pages 1173-1180 - Reply to Woiciechowsky. Acta Neurochirurgica 2007; 149(2): 213.

[7] Dvorak MF, Kwon BK, Fisher CG, Eiserloh HL, III, Boyd M, Wing PC. Effectiveness of titanium mesh cylindrical cages in anterior column reconstruction after thoracic and lumbar vertebral body resection. Spine (Phila Pa 1976 ) 2003; 28(9): 902-8.

[8] Cobos JA, Lindsey RW, Gugala Z. The cylindrical titanium mesh cage for treatment of a long bone segmental defect: description of a new technique and report of two cases. J Orthop Trauma 2000; 14(1): 54-9.

[9] Fujibayashi S, Kim HM, Neo M, Uchida M, Kokubo T, Nakamura T. Repair of segmental long bone defect in rabbit femur using bioactive titanium cylindrical mesh cage. Biomaterials 2003; 24(20): 3445-51.

[10] Lindsey RW, Gugala Z, Milne E, Sun M, Gannon FH, Latta LL. The efficacy of cylindrical titanium mesh cage for the reconstruction of a critical-size canine segmental femoral diaphyseal defect. J Orthop Res 2006; 24(7): 1438-53.

[11] Attias N, Lehman RE, Bodell LS, Lindsey RW. Surgical management of a long segmental defect of the humerus using a cylindrical titanium mesh cage and plates: a case report. J Orthop Trauma 2005; 19(3): 211-6.

[12] Attias N, Lindsey RW. Case reports: management of large segmental tibial defects using a cylindrical mesh cage. Clin Orthop Relat Res 2006; 450: 25966.

[13] Ostermann PA, Haase N, Rubberdt A, Wich M, Ekkernkamp A. Management of a long segmental defect at the proximal meta-diaphyseal junction of the tibia using a cylindrical titanium mesh cage. J Orthop Trauma 2002; 16(8): 597-601.

[14] Hasegawa K, Abe M, Washio T, Hara T. An experimental study on the interface strength between titanium mesh cage and vertebra in reference to vertebral bone mineral density. Spine (Phila $\mathrm{Pa}$ 1976) 2001; 26(8): 957-63.

[15] Chen Y, Chen D, Guo Y, Wang X, Lu X, He Z, Yuan W. Subsidence of titanium mesh cage: a study based on 300 cases. J Spinal Disord Tech 2008; 21(7): 489-92.

[16] Klezl Z, Bagley CA, Bookland MJ, Wolinsky JP, Rezek Z, Gokaslan ZL. Harms titanium mesh cage fracture. Eur Spine J 2007; 16 Suppl 3: 306-10.

[17] Ohman C, Baleani M, Pani C, Taddei F, Alberghini M, Viceconti M, Manfrini M. Compressive behaviour of child and adult cortical bone. Bone 2011; 49(4): 769-76.

[18] Vadapalli S, Sairyo K, Goel VK, Robon M, Biyani A, Khandha A, Ebraheim NA. Biomechanical rationale for using polyetheretherketone (PEEK) spacers for lumbar interbody fusion-A finite element study. Spine (Phila Pa 1976 ) 2006; 31(26): E992E998. 